\title{
Incidencia de complicaciones en cirugía dermatológica de cáncer de piel melanoma y no melanoma en pacientes con múltiple comorbilidad 0 antiagregantes-anticoagulantes. Experiencia de nuestro hospital durante 5 años
}

\author{
Incidence of complications in dermatological surgery of melanoma and non-melanoma \\ skin cancer in patients with multiple comorbidity and/or antiplatelet-anticoagulants. \\ Five year experience in our Hospital.
}

Lilia Arguello-Guerra, Estefanía Vargas-Chandomid, Jose Manuel Díaz-González, Silvia Méndez-Flores, Ana Ruelas-Villavicencio y Judith Domínguez-Cherit*

Departamento de Dermatología, Instituto Nacional de Ciencias Médicas y Nutrición Salvador Zubirán, Ciudad de México, México

\begin{abstract}
Resumen
Introducción: La cirugía es uno de los procedimientos que se realizan con mayor frecuencia en dermatología debido a la mayor incidencia de cáncer de piel melanoma y no melanoma. Se han encontrado distintas posturas entre los cirujanos dermatólogos sobre continuar o suspender antiagregantes y anticoagulantes antes del procedimiento, lo cual incrementa el riesgo de eventos tromboembólicos, además de la preferencia de utilizar profilaxis antibiótica de forma posquirúrgica por algunos dermatólogos. Objetivo: Reportar nuestra experiencia en cuanto a la seguridad de la cirugía dermatológica sin la suspensión de anticoagulantes/antiagregantes y sin profilaxis antibiótica en pacientes con múltiple comorbilidad y polifarmacia. Método: Se revisaron 655 pacientes. El 96.6\% tenían al menos otra enfermedad. El 27.7\% utilizaba ácido acetilsalicílico y el 4.3\% algún tipo de anticoagulante. El tipo de neoplasia más frecuente fue el carcinoma basocelular con 69.8\%. Resultados: La tasa total de complicaciones fue del 4.2\%. La complicación más frecuente fue la dehiscencia de la herida (1.1\%), seguida de la necrosis parcial (0.9\%), la infección (0.9\%), la reacción a cuerpo extraño (0.6\%), la necrosis total (0.3\%), la hemorragia (0.2\%) y la fístula cutánea (0.2\%). Conclusiones: Basándonos en la literatura y nuestra experiencia, la cirugía dermatológica es segura sin suspender antitrombóticos ni indicar profilaxis antibiótica en pacientes con múltiple comorbilidad.
\end{abstract}

PALABRAS CLAVE: Cirugía dermatológica. Dermatología oncológica. Complicaciones posquirúrgicas. Profilaxis antibiótica.

\begin{abstract}
Introduction: Surgery is performed more frequently now at days, due to the increasing incidence of melanoma and no-melanoma skin cancer. There are different opinions among dermatologic surgeons between to continue or discontinue antithrombotic therapy prior to the procedure, which increases the risk of thromboembolic events. Prophylaxis with oral antibiotics in the postsurgical period is controversial. Objective: To report the safety of surgery without suspending antithrombotic therapy and without oral antibiotic prophylaxis in dermatology surgery of patients with multiple comorbidities and polypharmacy. Method: We designed a retrospective study. We included a total of 655 patients; $96.6 \%$ had at least one comorbidity; $27.7 \%$ used aspirin
\end{abstract}

\author{
Correspondencia: \\ *Judith Domínguez-Cherit \\ Vasco de Quiroga, 15 \\ Col. Sección XVI, Del. Tlalpan \\ C.P. 14080, Ciudad de México, México \\ E-mail: judom59@hotmail.com
}

Fecha de recepción: 08-12-2016

Fecha de aceptación: 14-02-2018

DOI://dx.doi.org/10.24875/CIRU.M18000012
Cir Cir. 2018;86:20-28

Disponible en PubMed www.cirugiaycirujanos.com 
and $4.3 \%$ some type of antithrombotic therapy. The most common type of skin tumor was basal cell carcinoma with $69.8 \%$. Results: The complication rate was $4.2 \%$; the most was wound dehiscence (1.1\%), followed by partial necrosis (0.9\%), infection $(0.9 \%)$, reaction to foreign body $(0.6 \%)$, complete necrosis $(0.3 \%)$, bleeding $(0.2 \%)$ and fistulae $(0.2 \%)$. Conclusions: Based on the literature and our experience, dermatologic surgery is safe without suspending antithrombotic therapy or antibiotic prophylaxis in patients with multiple comorbidity.

KEY WORDS: Dermatologic surgery. Skin cancer. Skin surgery complications. Antibiotic prophylaxis.

\section{Introducción}

La cirugía es uno de los procedimientos que se realizan con mayor frecuencia en dermatología, debido al incremento en la incidencia de cáncer de piel melanoma y no melanoma ${ }^{1,2}$. Se realizan desde procedimientos superficiales, como rasurado, curetaje y crioterapia, hasta algunos de mayor complejidad, como es el caso de los colgajos y de los injertos cutáneos ${ }^{3}$.

La mayoría de los cirujanos dermatólogos tienen diferentes posturas en cuanto a la suspensión de los medicamentos antitrombóticos durante la cirugía dermatológica. Algunos optan por la suspensión una semana previa al procedimiento, mientras otros mantienen el fármaco antitrombótico perioperatorio ${ }^{4-7}$. El riesgo por suspensión y su asociación con eventos trombóticos es mayor que el beneficio de suspenderlos ${ }^{6}$.

De forma general, entre las complicaciones más frecuentes de la cirugía dermatológica que se han reportado se encuentran la infección del sitio de la herida, la pérdida del injerto o colgajo, la hemorragia y los defectos en el cierre ${ }^{8}$. La complicación más frecuente asociada al uso de antitrombóticos es la hemorragia ${ }^{4-7}$.

Actualmente, el uso desproporcionado de antibióticos ocasiona gastos al sistema de salud, además de exposición a efectos adversos e incremento en las resistencias bacterianas ${ }^{9}$.

\section{Objetivo}

Reportar la seguridad de la cirugía dermatológica sin la suspensión de anticoagulantes/antiagregantes y sin el uso de antibióticos de forma profiláctica en pacientes con comorbilidad y polifarmacia en nuestro Instituto.

\section{Desenlaces}

- Demostrar la baja tasa de complicaciones de forma global, en comparación con estudios similares previos.
- Demostrar la baja tasa de infecciones sin el uso de antibiótico profiláctico.

- Demostrar la baja tasa de incidencia de episodios hemorrágicos sin suspender los tratamientos antitrombóticos.

- Demostrar la baja tasa de complicaciones en colgajos e injertos.

- Demostrar la baja tasa de complicaciones asociadas al tabaquismo.

\section{Método}

\section{Diseño del estudio}

- Estudio retrospectivo.

- Cinco años, desde enero de 2011 hasta diciembre de 2015.

- Un solo centro.

\section{Pacientes}

Los criterios de inclusión fueron:

- Pacientes con diagnóstico de cáncer de piel melanoma y no melanoma a quienes se realizó algún procedimiento quirúrgico del 1 de enero de 2011 al 31 de diciembre de 2015.

- Solo procedimientos realizados por el servicio de dermatología.

- Procedimientos realizados de forma ambulatoria.

Los criterios de exclusión fueron:

- Pacientes con expediente incompleto.

- Pacientes sin registro en el Instituto.

- Pacientes sin confirmación histológica.

- Pacientes que no contaran con revisión posquir úrgica.

- Procedimientos realizados por personal de otras especialidades.

\section{Análisis estadístico}

Se definieron los procedimientos que realizamos en nuestro centro como cirugía limpia de acuerdo con 
Ios criterios de los Centers for Disease Control and Prevention $(C D C)^{10}$ :

- Se consideraron, según la histología, para riesgo bajo, medio y alto las guías de la National Comprehensive Cancer Network (NCCN) para cáncer de piel melanoma y no melanoma ${ }^{11-13}$.

- Se clasificó, por topografía, de bajo y alto riesgo según las guías de la NCCN ${ }^{11-13}$.

- No se consideró el tamaño del tumor ni del defecto quirúrgico.

- No se utilizó antibiótico de manera profiláctica.

- Los datos considerados fueron edad, tabaquismo, comorbilidad, uso de fármacos antitrombóticos, tipo de cáncer de piel, localización, y presencia o ausencia de complicaciones posquirúrgicas.

En la mayoría de los pacientes se realizó escisión con hoja de bisturí del No. 15 con distintos tipos de cierre:

- Cierre directo.

- Colgajo de avance.

- Colgajo de rotación.

- Colgajo de transposición.

- Injerto.

- Cierre por segunda intención.

En cuanto a las complicaciones, se definieron como:

- Hemorragia: presencia de hematoma o persistencia de sangrado en las 24 horas posteriores al procedimiento, que requirieran reintervención para ligar un vaso sangrante o para drenaje de hematoma ${ }^{14}$.

- Infección: presencia de exudado purulento, incremento de temperatura local, eritema perilesional y dolor progresivo en las primeras 72 horas posquirúrgicas.

- Necrosis de colgajo e injerto: parcial (necrosis hasta del $75 \%$ ) o total (necrosis mayor del $75 \%$ ).

- Dehiscencia: abertura espontánea de la herida afrontada con sutura, sin presencia de necrosis del tejido.

- Eritema o reacción a cuerpo extraño.

Estos eventos se documentaron en el expediente durante la revisión posquirúrgica habitual a los 7-14 días (antes de retirar la sutura, durante el retiro 0 posterior al retiro).

\section{Resultados}

En la tabla 1 se observan las características basales de nuestra población. Se valoraron 655 pacientes, de los cuales el $50.1 \%(n=328)$ eran mujeres y el
49.9\% $(n=327)$ eran hombres; la edad promedio era de 74 años. En la población de estudio, el $96.6 \%$ $(n=633)$ tenían diagnóstico de al menos otra enfermedad, con un máximo de 12 y una media de tres; de ellas, las más comunes fueron hipertensión arterial sistémica $(48.1 \% ; n=315)$, diabetes mellitus tipo $2(29.8 \% ; n=195)$, dislipidemia $(26.4 \% ; n=173)$, neoplasia de órgano sólido y hematológica (26.1\%; $n$ $=171)$, eventos cardiovasculares $(18.9 \% ; n=124)$, inmunosupresión por fármacos o por infección por el virus de la inmunodeficiencia humana (10.1\%; $n=66)$, y trasplante de órgano sólido $(7 \% ; n=46)$. La presencia de tabaquismo al momento de la cirugía se documentó en el $12.8 \%(n=84)$ de los pacientes.

El número de medicamentos reportados al momento de la cirugía fueron desde 0 hasta 11 por paciente (promedio de 3) (Tabla 2). El antiagregante más frecuente fue el ácido acetilsalicílico (AAS) (27.2\%; $n=177)$, seguido del clopidogrel $(1.2 \% ; n=8)$. Los anticoagulantes se reportaron en el $4.3 \%(n=28)$ de

Tabla 1. Características basales de la población

\begin{tabular}{lc}
\hline Característica & $\%$ (n) \\
\hline Total pacientes & $100(655)$ \\
Hombres & $49.9(327)$ \\
Mujeres & $50.1(328)$ \\
Edad promedio (intervalo) & 74 años (20-101) \\
Sanos & $3.4(22)$ \\
Comorbilidad & $96.6(633)$ \\
No comorbilidad, promedio (intervalo) & $3(0-12)$ \\
Diabetes mellitus tipo 2 & $29.8(195)$ \\
Hipertensión arterial sistémica (HAS) & $48.1(315)$ \\
Dislipidemia (DLP) & $26.4(173)$ \\
Neoplasia & $26.1(171)$ \\
Cardiovascular & $18.9(124)$ \\
Inflamatoria & $3.4(22)$ \\
Reumatológica & $7(46)$ \\
Inmunosupresión & $10.1(66)$ \\
Trasplante & $7(46)$ \\
Tabaquismo & $12.8(84)$ \\
\hline
\end{tabular}

Tabla 2. Descripción de los medicamentos totales por paciente. Antiagregantes y anticoagulantes utilizados durante la cirugía dermatológica

\begin{tabular}{lc}
\hline Medicamentos & $\%(\mathbf{n})$ \\
\hline Medicamentos promedio por & \\
paciente & $3(0-11)$ \\
Antiagregantes & $28.1(184)$ \\
Ácido acetilsalicílico & $27.2(177)$ \\
Clopidogrel & $1.2(8)$ \\
Anticoagulantes & $4.3(28)$ \\
Acenocumarina & $2.6(17)$ \\
Warfarina & $0.5(3)$ \\
Apixabán & $0.5(3)$ \\
Enoxaparina & $0.3(2)$ \\
Dabigatrán & $0.3(2)$ \\
Rivaroxabán & $0.2(1)$ \\
\hline
\end{tabular}


los pacientes, con la acenocumarina como el más frecuente $(2.6 \% ; n=17)$.

El tipo de tumor que predominó fue el carcinoma basocelular de bajo $(24 \% ; n=157)$ y alto grado (45.8\%; $n=300)$; el resto se distribuyó entre carcinoma epidermoide (CEC) in situ (13.3\%; $n=87$ ), CEC bien diferenciado $(9.3 \% ; n=61)$, CEC mal diferenciado $(0.2 \% ; n=1)$, melanoma in situ $(2.6 \% ; n=17)$, melanoma invasor $(3.4 \% ; n=22)$, porocarcinoma $(1.1 \% ; n=7)$, siringocistadenocarcioma $(0.2 \% ; n=1)$, carcinoma triquilémico $(0.2 \% ; n=1)$ y dermatofibrosarcoma protuberans $(0.2 \% ; n=1$ ) (Tabla 3$)$. De igual forma, la mayoría de los pacientes, por topografía, se ubicaron en zonas de alto riesgo (56.5\%) de acuerdo con la clasificación de la NCCN.

En los 655 pacientes se realizó escisión, en la mayoría $(99.1 \%)$ con margen de al menos $5 \mathrm{~mm}$; en el resto no se completó la ampliación de márgenes debido a que tras la revisión posquirúrgica no acudieron a un segundo tiempo quirúrgico. Los tipos de cierre que más se utilizaron fueron el cierre directo $(82.9 \%$; $\mathrm{n}=543$ ), seguido de colgajos de distintos tipos, como colgajo de avance (4.3\%; $n=28)$ (Fig. 1), de rotación $(3.7 \% ; n=24)$ (Figs. 2 y 3 ) y de transposición (4.9\%; $n=11)$, el injerto $(4.9 \% ; n=32)$ y el cierre por segunda intención (2.6\%; $n=17)$. En cuanto a las complicaciones, se reporta un $4.2 \%(n=27)$ y en orden de frecuencia fueron dehiscencia de la herida $(1.1 \% ; n=7)$, necrosis parcial $(0.9 \% ; n=6)$, infección $(0.9 \% ; n=6)$, reacción a cuerpo extraño $(0.6 \% ; n=4)$, necrosis total $(0.3 \% ; n=2)$, hemorragia $(0.2 \% ; n=1)$ y fístula cutánea $(0.2 \% ; n=1)$ (Tabla 4$)$.

De los 27 pacientes en que se documentó alguna complicación (Tabla 5), el único paciente con reporte de hemorragia tenía 72 años, se presentó en el dorso nasal, no utilizaba antitrombóticos ni tabaco, y solo contaba con una comorbilidad. En seis pacientes se encontró necrosis parcial, de los cuales tres se registraron en el dorso nasal (dos con colgajo de rotación y uno con injerto), y solo uno de ellos con uso de anticoagulante; uno fue con cierre directo en piel cabelluda, otro en la pierna izquierda y el otro con colgajo de rotación en la mejilla izquierda. De los dos pacientes con necrosis total de colgajo, uno se documentó en el dorso nasal con uso de AAS y el otro en la hélix izquierda con injerto. En cuanto al sitio de infección, se observaron dos en la piel cabelluda, dos en el brazo y uno en la boca y el área genital. Solo un paciente con cirugía en el brazo utilizaba AAS. La dehiscencia se presentó en siete pacientes, de los cuales hubo dos en el tronco y uno de cada uno en la mejilla, la pierna, la oreja, la
Tabla 3. Tipo de tumores resecados. Topografía por bajo riesgo y alto riesgo

\begin{tabular}{lc}
\hline Tipo de tumores & $\%(\mathbf{n})$ \\
\hline Total tumores & $100(655)$ \\
CBC bajo riesgo & $24(157)$ \\
CBC alto riesgo & $45.8(300)$ \\
CEC in situ & $13.3(87)$ \\
CEC bien diferenciado & $9.3(61)$ \\
CEC mal diferenciado & $0.2(1)$ \\
Melanoma in situ & $2.6(17)$ \\
Melanoma invasor & $3.4(22)$ \\
Porocarcinoma & $1.1(7)$ \\
Siringocistadenocarcinoma & $0.2(1)$ \\
Cáncer triquilémico & $0.2(1)$ \\
Dermatofibrosarcoma protuberans & $0.2(1)$ \\
Topografía de bajo riesgo & $43.5(285)$ \\
Topografía de alto riesgo & $56.5(370)$ \\
\hline
\end{tabular}

CBC: carcinoma basocelular; CEC: carcinoma epidermoide

Tabla 4. Tipo de tratamiento quirúrgico, tipo de cierre y tipo de complicaciones

\begin{tabular}{lc}
\hline Tratamiento quirúrgico & $\%$ (n) \\
\hline Escisional & $100(655)$ \\
Sin margen & $0.9(6)$ \\
Con margen & $99.1(649)$ \\
Tipo de cierre & \\
Directo & $82.9(543)$ \\
Colgajo de avance & $4.3(28)$ \\
Colgajo de rotación & $3.7(24)$ \\
Colgajo de transposición & $1.7(11)$ \\
Injerto & $4.9(32)$ \\
Segunda intención & $2.6(17)$ \\
Complicaciones & $4.2(27)$ \\
Tipo de complicación & \\
Hemorragia & $0.2(1)$ \\
Necrosis parcial & $0.9(6)$ \\
Necrosis total & $0.3(2)$ \\
Infección & $0.9(6)$ \\
Dehiscencia & $1.1(7)$ \\
Reacción a cuerpo extraño & $0.6(4)$ \\
Fístula cutánea & $0.2(1)$ \\
\hline
\end{tabular}

región temporal y el mentón. De estos, dos pacientes (región temporal y oreja) utilizaban AAS. La reacción a cuerpo extraño se observó en dos pacientes, uno en el tronco y otro en la pierna; este último utilizaba AAS.

\section{Discusión}

Los resultados de esta revisión concuerdan con los previamente reportados en estudios similares ${ }^{15}$. La mayoría de los procedimientos se realizaron por cirujanos dermatólogos y residentes de dermatología. No se realizó cirugía micrográfica de Mohs (CMM). Como parte de nuestro protocolo, no se suspendieron los anticoagulantes ni los antiagregantes en ningún procedimiento, según las recomendaciones de la Asociación Americana del Corazón, y tampoco se indicaron antimicrobianos profilácticos sistemáticos ${ }^{16}$. 


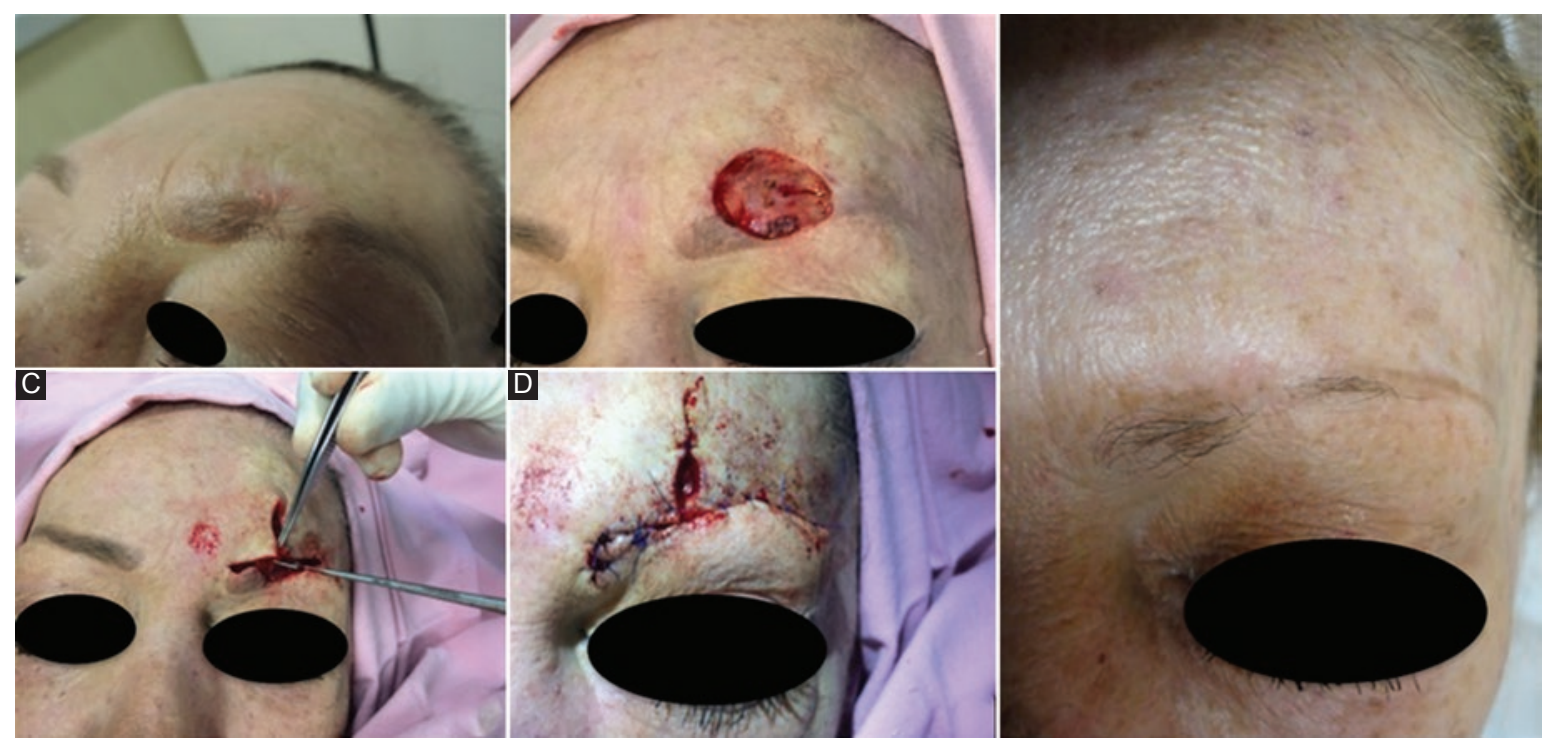

Figura 1. A: carcinoma basocelular micronodular supraciliar izquierdo. B: defecto primario. C y D: colgajo de avance "O en T». E: seguimiento a 1 año.

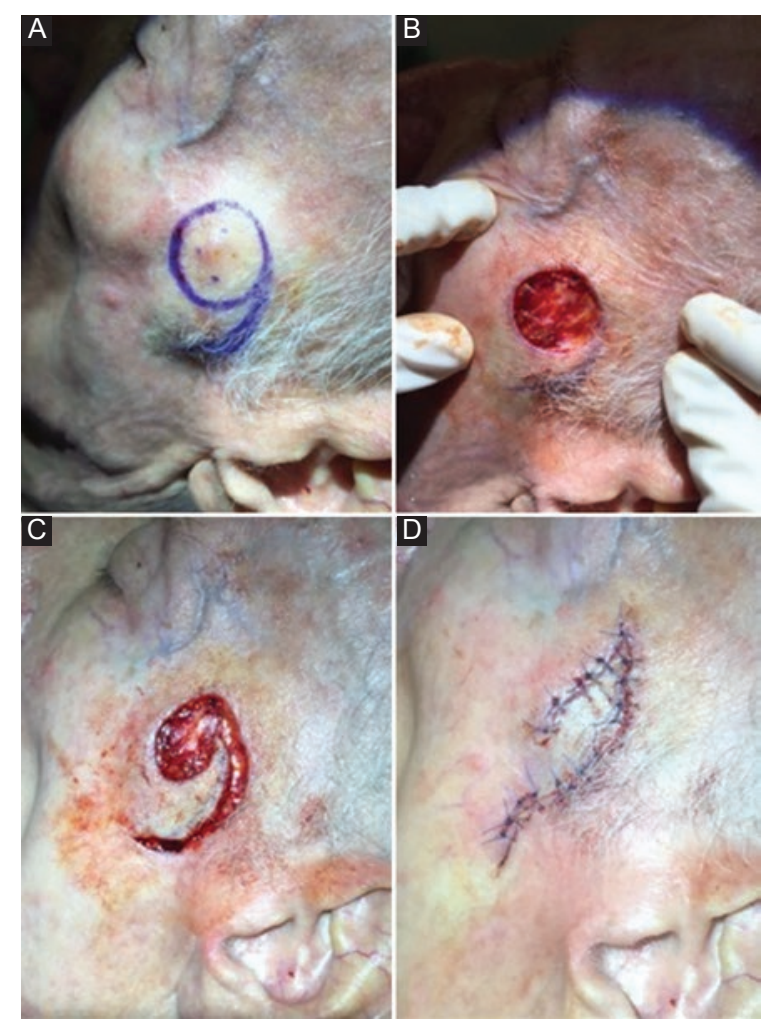

Figura 2. A: carcinoma basocelular nodular. B: defecto primario. C: colgajo de rotación. D: cierre del colgajo de rotación.

Actualmente, la incidencia del cáncer de piel melanoma y no melanoma se encuentra en aumento ${ }^{1,2}$. Los pacientes, a mayor edad tienen más comorbilidad, y por lo mismo requieren el uso de numerosos medicamentos, incluyendo aquellos con efecto antitrombótico y anticoagulante ${ }^{17,18}$.
La tasa global de complicaciones registrada fue similar a la de reportes previos. El primer estudio que comparó las complicaciones posquirúrgicas en CMM en pacientes que utilizaban antitrombóticos y antiinflamatorios no esteroideos fue el de Otley, et al. ${ }^{19}$, incluyendo hemorragia, dehiscencia y necrosis de colgajos e injertos, reportando una tasa del $4.58 \%$, similar a la nuestra del $4.2 \%$.

Infección se considera a la presencia de exudado purulento además de un dato clínico como fiebre, eritema, edema, calor o induración presentes en los primeros 4-7 días tras el procedimiento quirúrgico ${ }^{10,20}$.

En nuestro centro no se utilizan sistemáticamente antibióticos de forma profiláctica en este tipo de procedimientos, de acuerdo con las guías de la Asociación Americana del Corazón ${ }^{16}$ y la Academia Americana de Cirujanos Ortopedistas, en las que se establecen los procedimientos y sitios anatómicos de alto riesgo para el desarrollo de infección.

Los CDC consideran la cirugía dermatológica como una cirugía limpia, y calculan que sin presencia de inflamación y con técnica estéril tiene un riesgo de infección inferior al $5 \%{ }^{10}$. Comparada nuestra tasa de infección del $0.9 \%$ con las cifras reportadas en otros estudios (Tabla 6), podemos observar que no difiere de la observada con el uso de profilaxis antimicrobiana, especialmente con el estudio de Maragh y Brown ${ }^{21}$, que analizaron 1115 tumores con CMM sin el uso de profilaxis antimicrobiana, obteniendo una tasa de infección del $0.7 \%$. 
Tabla 5. Complicaciones posquirúrgicas por topografía, tipo de cierre, tabaquismo y uso de antitrombóticos

\begin{tabular}{|c|c|c|c|c|c|}
\hline Complicaciones & $\%(n)$ & Topografía & Tipo de cierre & Tabaco & Antitrombótico \\
\hline Total & $4.2(27)$ & & & & \\
\hline \multicolumn{6}{|l|}{ Tipo de complicación } \\
\hline Hemorragia & $0.2(1)$ & DN & $C D$ & No & No \\
\hline \multirow[t]{6}{*}{ Necrosis parcial } & $0.9(6)$ & $\mathrm{PC}$ & CD & No & No \\
\hline & & PD & $\mathrm{CA}$ & No & No \\
\hline & & $\mathrm{Ml}$ & INJ & No & Sí \\
\hline & & DN & $\mathrm{CR}$ & No & No \\
\hline & & DN & $\mathrm{CR}$ & Sí & No \\
\hline & & DN & $\mathrm{CR}$ & No & Sí \\
\hline \multirow[t]{2}{*}{ Necrosis total } & $0.3(2)$ & DN & CA & No & Sí \\
\hline & & $\mathrm{Ol}$ & INJ & Sí & No \\
\hline \multirow[t]{6}{*}{ Infección } & $0.9(6)$ & $\mathrm{PC}$ & CD & No & No \\
\hline & & PC & $C D$ & No & No \\
\hline & & LD & $C D$ & No & No \\
\hline & & LMI & $C D$ & No & No \\
\hline & & $\mathrm{Bl}$ & $C D$ & No & Sí \\
\hline & & PD & $C D$ & No & No \\
\hline \multirow[t]{7}{*}{ Dehiscencia } & $1.1(7)$ & $\mathrm{Ml}$ & CD & Sí & Sí \\
\hline & & TA & CD & No & No \\
\hline & & TP & $C D$ & No & No \\
\hline & & PI & $C D$ & Sí & Sí \\
\hline & & Ol & CSI & No & Sí \\
\hline & & TD & CT & No & Sí \\
\hline & & M & $C D$ & No & No \\
\hline \multirow[t]{4}{*}{ Reacción a cuerpo extraño } & $0.6(4)$ & PD & CD & No & No \\
\hline & & $\mathrm{Ml}$ & CD & No & No \\
\hline & & MD & $C D$ & Sí & No \\
\hline & & $\mathrm{TA}$ & $C D$ & No & No \\
\hline Fístula cutánea & $0.2(1)$ & MD & $C D$ & No & No \\
\hline
\end{tabular}

CA: colgajo de avance; CD: cierre directo; CR: colgajo de rotación; CSI: cierre segunda intención; CT: colgajo de transposición; DN: dorso nasal; INJ: injerto; LD: labio derecho; LMI: labio mayor izquierdo; M: mentón; MD: mejilla derecha; MI: mejilla izquierda; OI: oreja izquierda; PC: piel cabelluda; PD: pierna derecha; PI: pierna izquierda; TA: tronco anterior; TD: temporal derecho; TP: tronco posterior.

Las cirugías que requieren profilaxis antimicrobiana son las que se realizan sobre mucosas y en sitios con infección activa en el momento del procedimiento.

Por topografía, la mayor tasa de complicaciones se ha reportado en los labios, las orejas, la región inguinal y en injertos y colgajos en la nariz, alcanzando hasta un $5 \%^{22,23}$.

En un estudio más reciente con 69 pacientes se reporta que, por debajo de la rodilla, aparecieron hasta un $17 \%$ de complicaciones, destacando que el $8.9 \%$ del total se infectaron, mientras que en el resto se observó retraso en la cicatrización, hipergranulación y cicatrización deficiente ${ }^{24}$.

En nuestro estudio, los pacientes que presentaron infección fueron aquellos que tuvieron cirugía en piel cabelluda (dos pacientes), uno en boca, uno en el área genital y dos en el brazo.
La comorbilidad que incrementa el riesgo de infección incluye la inmunosupresión farmacológica, la diabetes y el tabaquismo; sin embargo, Dixon ${ }^{23}$ no logró demostrar una diferencia significativa entre fumadores y no fumadores y sus complicaciones. Por el contrario, Wahie y Lawrence ${ }^{25}$ reportan que la tasa de complicaciones en heridas fue significativamente más alta en fumadores que en no fumadores: $63 \%$ vs. $12 \%$, respectivamente.

La mayoría de los autores recomiendan personalizar el uso de profilaxis según el procedimiento y la topografía, utilizando una cefalosporina de primera generación (previo a la cirugía). Cabe señalar que es una recomendación basada en procedimientos de otras especialidades ${ }^{9}$. Levender, et al. ${ }^{26}$ analizaron retrospectivamente el uso de antibióticos tópicos como profilaxis en procedimientos limpios en 
Tabla 6. Incidencia de infecciones ${ }^{9}$

\begin{tabular}{llcc}
\hline Autor, año & Tipo de cirugía & Casos (N) & Tasa de infección (\%) \\
\hline Maragh, 2008 ${ }^{21}$ & CMM & 1115 & 0.7 \\
Tanna, 2008 & Ritidectomías de cicatrices & 1000 & 0.0 \\
Dixon, 2006 & CPNM (varias técnicas), total: & 5091 & 1.47 \\
& Curetaje & $3 / 412$ & \\
& Colgajos & $47 / 1601$ & \\
& Injertos & $6 / 69$ & 1.78 \\
Rhinehart, 2006 & Escisiones & $16 / 2974$ & 2.29 \\
Futoryan, 1995 & Ampliaciones & $3 / 35$ & 2.29 \\
Cook, 2003 & CMM guantes estériles vs. no estériles & 1400 & 0.07 \\
Kimyai-Asadi, 2005 & Escisión & 530 & 1.9 \\
\hline
\end{tabular}

CMM: cirugía micrográfica de Mohs; CPNM: cáncer de piel no melanoma.

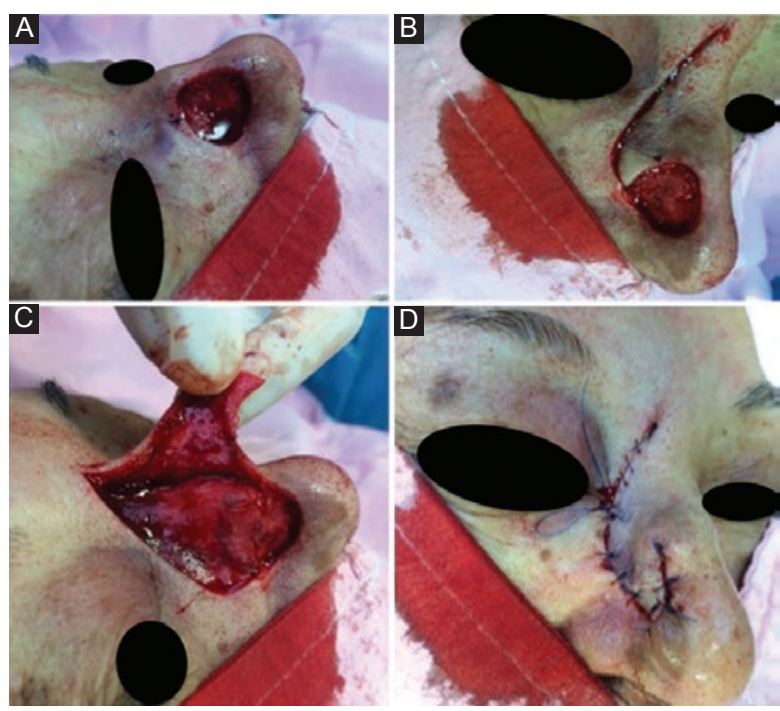

Figura 3. A: defecto primario de carcinoma basocelular nodular. B, C y D: colgajo de rotación.

dermatología realizados por dermatólogos y médicos no dermatólogos, y observaron que el $6.0 \%$ y el $3.5 \%$, respectivamente, utilizan antimicrobiano tópico, sin diferencia en el desenlace, por lo que proponen detener su utilización de forma excesiva.

Los resultados de nuestro estudio apoyan las recomendaciones de no utilizar tratamiento antibiótico profiláctico de manera preventiva, excepto en casos especiales, como antecedente de prótesis cardiaca y articular, y en cirugía en sitios de alto riesgo de infección.

Encuestas aplicadas a médicos que realizan cirugía de piel reportan que hasta el $50 \%$ suspenden siempre los medicamentos antitrombóticos antes del procedimiento ${ }^{5,27}$. En nuestro estudio, el $28.1 \%$ de los pacientes utilizaban antiagregantes y el $4.3 \%$ algún tipo de anticoagulación, mismos que continuaron durante el procedimiento (Tabla 2). Solo se reportó un paciente con hemorragia $(0.2 \%)$ sin antecedente de terapia antitrombótica, porcentaje más bajo que el referido en el estudio de Shimizu, et al. ${ }^{28}$, en el cual solo cuatro pacientes $(0.53 \%)$ de 760 presentaron hemorragia, resultado similar al del estudio de Shipkov, et al..$^{14}$, con un solo paciente con hemorragia y uso de anticoagulante. Bordeaux, et al. ${ }^{29}$, en su estudio, reportan un $0.89 \%$ de complicaciones hemorrágicas, la mayoría asociadas con el uso de anticoagulantes.

En nuestro estudio, solo nueve de los 27 pacientes con complicaciones utilizaron AAS, y uno acenocumarina. Dentro de las complicaciones que se presentaron están la necrosis parcial y total en tres pacientes, la infección en dos pacientes, la dehiscencia en cuatro pacientes y la reacción a cuerpo extraño en un paciente.

Se han realizado pocos estudios en cirugía dermatológica comparando el uso de este tipo de medicamentos; sin embargo, en cirugías de mayor complejidad sí se establece la suspensión en días previos. Algunos estudios prospectivos que se han realizado demostraron una baja tasa de complicaciones. Por ejemplo, uno de los primeros estudios fue el de Bartlett $^{30}$, que comparó a 52 pacientes tomando AAS y 119 pacientes sin AAS, obteniendo un total del $5.7 \%$ frente al $7.5 \%$ de complicaciones, respectivamente, por lo que concluye que no se deberá suspender su uso para este tipo de procedimientos. En nuestro estudio el resultado fue menor, ya que solo encontramos un $5 \%$ de complicaciones en el total de pacientes con uso de AAS (9 de 177).

Al igual que en estudios previos, no se observó un incremento en el riesgo de hemorragia en los pacientes con $\mathrm{AAS}^{18,28-34}$, y en cambio sí se han demostrado complicaciones trombóticas al suspender este tipo de medicamentos ${ }^{35,36}$. 
Podemos afirmar, al menos en nuestro estudio, que las complicaciones hemorrágicas en los pacientes con antiagregantes y anticoagulantes están ausentes, mismos hallazgos que los reportados por Alcalay ${ }^{37}$.

En nuestro estudio, del 100\% ( $n=84)$ de los fumadores, solo el $5.95 \%(n=5)$ presentaron algún tipo de complicación: necrosis parcial de colgajo de nariz, necrosis total de colgajo en oreja y dos dehiscencias, una en la pierna y otra en la mejilla, además de una reacción a cuerpo extraño en la mejilla que se atribuye a la presencia de sutura.

En relación con el tabaco, en colgajos e injertos es importante documentar el número de paquetes por día (PPD) consumidos, ya que se ha establecido una relación dosis-respuesta entre los PPD y la presencia de necrosis. Goldminz y Bennett ${ }^{38}$ revisaron una serie de 916 colgajos e injertos de piel de espesor total y encontraron una relación dosis-respuesta significativa entre los PPD y el desarrollo de necrosis: los fumadores de un PPD tuvieron dos veces mayor riesgo de necrosis de injerto, y aquellos con dos PPD tuvieron seis veces mayor riesgo de desarrollar necrosis comparados con la población de no fumadores, de fumadores de menos de un PPD y de exfumadores ${ }^{38}$. No hay un consenso ni guías que establezcan el intervalo de tiempo para el cese del tabaco previo a la cirugía; la mayoría de los estudios recomienda de 1 día a 4 semanas antes de la cirugía, reanudando entre los 5 días y hasta 4 semanas del posoperatorio ${ }^{39}$. Sin embargo, en aquellos pacientes con tabaquismo intenso (más de un PPD), solo el $23 \%$ logró suspender por completo su consumo durante 4 semanas $^{40}$, por lo que se sugiere disminuir su consumo a menos de un PPD por lo menos 1 semana antes de la cirugía y 1 semana después ${ }^{38}$.

Por lo tanto, en este tipo de pacientes se recomienda advertir acerca de las posibles complicaciones de la herida causadas por el tabaquismo, y se debe indicar la suspensión del tabaco por lo menos 2 semanas antes y reanudar 1 semana después de la cirugía, en las que pueden utilizarse terapias de sustitución de nicotina. En los pacientes con tabaquismo intenso que se niegan a suspender el hábito se recomienda indicarles un consumo menor de un PPD ${ }^{39}$.

La necrosis parcial de los colgajos e injertos ocurrió en el $0.9 \%$ de los realizados (6/95), y la necrosis total en el $0.3 \%(n=2)$. El tejido necrótico se retiró y se dejó a cierre de segunda intención. Los sitios anatómicos fueron piel cabelluda, extremidades inferiores, mejilla, dorso nasal y oreja, lo cual es menor al compararlo con otros estudios, como el de Bordeaux, et al. ${ }^{29}$, con una tasa del 1.7\% de necrosis parcial (frente, párpado, nariz y mejillas), sin demostrar una relación entre sitio y necrosis, aunque sí predominó la necrosis parcial de injerto en la nariz y la piel cabelluda.

En cuanto a la dehiscencia, obtuvimos un $1.1 \%$ $(n=7)$, con predominio en la cara (nariz, región malar y temporal), porcentaje apenas superior a lo reportado en estudios similares con un tasa del $0.73 \%{ }^{21}$ y coincidiendo con los sitios anatómicos. Kimyai-Asadi, et al. ${ }^{41}$ demostraron resultados similares a los nuestros, aunque en CMM.

La evaluación preoperatoria estandarizada permite evaluar de forma anticipada las normativas a seguir en cada paciente ${ }^{42}$. Se debe documentar el estado de salud, así como prevenir las complicaciones según la comorbilidad y la ingesta de medicamentos, y valorar el riesgo posquirúrgico, tal como describe $\mathrm{Otley}^{43}$ en su propuesta de evaluación preoperatoria, de modo similar a lo que realizamos en nuestro centro.

\section{Limitaciones}

Fue un estudio retrospectivo, de un único centro y limitado a 5 años, por lo que la población fue de solo 655 pacientes. No se documentaron el número de cigarrillos consumidos al momento de la cirugía ni el tamaño del tumor o del defecto quirúrgico.

\section{Conclusiones}

Basados en la literatura y en nuestra experiencia, concluimos que la cirugía dermatológica en pacientes con comorbilidad y polifarmacia, incluyendo antiagregantes 0 anticoagulantes, presenta la misma tasa de complicaciones, por lo que no se recomienda la suspensión de ningún tipo de medicamento en procedimientos ambulatorios, debido a que supera el riesgo de un evento trombótico. De igual forma, la baja tasa de infecciones sin el uso de profilaxis fue similar a los reportes descritos, y por lo tanto no se recomienda el uso sistemático de antibióticos. La cirugía dermatológica ambulatoria es segura en este tipo de pacientes sin hacer modificaciones en los tratamientos establecidos.

\section{Responsabilidades éticas}

Protección de personas y animales. Los autores declaran que para esta investigación no se han realizado experimentos en seres humanos ni en animales. 
Confidencialidad de los datos. Los autores declaran que han seguido los protocolos de su centro de trabajo sobre la publicación de datos de pacientes.

Derecho a la privacidad y consentimiento informado. Los autores han obtenido el consentimiento informado de los pacientes y/o sujetos referidos en el artículo. Este documento obra en poder del autor de correspondencia

\section{Financiamiento}

No se recibio financiamento ni publico ni privado.

\section{Conflicto de intereses}

No existen conflictos de intereses por parte de ninguno de los autores.

\section{Bibliografía}

1. Universidad Nacional Autónoma de México. México: Boletín UNAMDGCS-362; c2016. (Actualizado el 26 de mayo de 2016; consultado el 11 de septiembre de 2016.) Disponible en: http://www.dgcs.unam.mx/ boletin/bdboletin/2016_362.html

2. American Cancer Society. Cancer Facts \& Figures 2016. Atlanta: American Cancer Society; 2016.

3. Roenigk RK. Dermatologists perform more skin surgery than any other specialist: implications for health care policy, graduate and continuing medical education. Dermatol Surg. 2008;34:293-300.

4. Alcalay J, Alkalay R. Controversies in perioperative management of blood thinnersin dermatologic surgery: continue or discontinue? Dermatol Surg. 2004;30:1091-4; discussion 1094

5. Kovich O, Otley CC. Perioperative management of anticoagulants and platelet inhibitors for cutaneous surgery: a survey of current practices. Dermatol Surg. 2002;28:513-7.

6. Otley CC. Continuation of medically necessary aspirin and warfarin during cutaneous surgery. Mayo Clin Proc. 2003;78:1392-6.

7. Callahan S, Goldsberry A, Kim H, Yoo S. The management of antithrombotic medication in skin surgery. Dermatol Surg. 2012;38:1417-26.

8. Council ML, Alam M, Gloster HM Jr, Bordeaux JS, Carroll BT, Leitenberger $\mathrm{JJ}$, et al. Identifying and defining complications of dermatologic surgery to betracked in the American College of Mohs Surgery (ACMS) Registry. J Am Acad Dermatol. 2016;74:739-45.

9. Shurman DL, Benedetto AV. Antimicrobials in dermatologic surgery: facts and controversies. Clin Dermatol. 2010;28:505-10.

10. Berríos-Torres SI. Evidence-based update to the U.S. Centers for Disease Control and Prevention and Healthcare Infection Control Practices Advisory Committee guideline for the prevention of surgical site infection: developmental process. Surg Infect (Larchmt). 2016;17:256-61.

11. Bichakjian CK, Olencki T, Aasi SZ, Alam M, Andersen JS, Berg D, et al. Basal cell skin cancer, version 1.2016, NCCN Clinical Practice Guidelines in Oncology. J Natl Compr Canc Netw. 2016;14:574-97.

12. Coit DG, Thompson JA, Algazi A, Andtbacka R, Bichakjian CK, Carson WE $3^{\text {rd }}$, et al. Melanoma, version 2.2016, NCCN Clinical Practice Guidelines in Oncology. J Natl Compr Canc Netw. 2016;14:450-73.

13. Bichakjian CK, Olencki T, Aasi SZ, Alam M, Andersen JS, Berg D, et al. Squamous Cell Skin Cancer, version 1.2016, NCCN Clinical Practice Guidelines in Oncology. J Natl Compr Canc Netw. 2016;14:574-97.

14. Shipkov H, Irthum $C$, Seguin $P$, Mojallal A, Braye F. Evaluation of the risk of post-operative bleeding complications in skin cancer surgery without interruption of anticoagulant/antithrombotic medication: a prospective cohort study. J Plast Surg Hand Surg. 2015;49:242-6.

15. Jarjis RD, Jørgensen L, Finnerup K, Birk-Sørensen L. Complications in skin grafts when continuing antithrombotic therapyprior to cutaneous surgery requiring skin grafting: a systematic review. J Plast Surg Hand Surg. 2015;49:129-34.

16. Wilson W, Taubert KA, Gewitz M, Lockhart PB, Baddour LM, Levison M, et al. Prevention of infective endocarditis: guidelines from the American Heart Association: a guideline from the American Heart Association Rheumatic Fever, Endocarditis and Kawasaki Disease Committee, Council on Cardiovascular Disease in the Young, and the Council on
Clinical Cardiology, Council on Cardiovascular Surgery and Anesthesia, and the Quality of Care and Outcomes Research Interdisciplinary Working Group. J Am Dent Assoc. 2008;139(Suppl):3S-24S. Erratum in: J Am Dent Assoc. 2008;139:253.

17. Harrington RA, Becker RC, Ezekowitz M, Meade TW, O'Connor CM, Vorchheimer DA, et al. Antithrombotic therapy for coronary artery disease: the Seventh ACCP Conference on Antithrombotic and Thrombolytic Therapy. Chest. 2004;126(3 Suppl):513-48S.

18. Sabatine MS, Cannon CP, Gibson CM, López-Sendón JL,Montalescot G, Theroux $P$, et al. Addition of clopidogrel to aspirin and fibrinolytic therapy for myocardial infarction with ST-segment elevation. N Engl J Med. 2005;352:1179-89.

19. Otley CC, Fewkes JL, Frank W, Olbricht SM. Complications of cutaneous surgery in patients who are taking warfarin, aspirin, or nonsteroidal anti-inflammatory drugs. Arch Dermatol. 1996;132:161-6.

20. Messingham MJ, Arpey CJ. Update on the use of antibiotics in cutaneous surgery. Dermatol Surg. 2005;31:1068-78.

21. Maragh SL, Brown MD. Prospective evaluation of surgical site infection rate among patients with Mohs micrographic surgery without the use of prophylactic antibiotics. J Am Acad Dermatol. 2008;59:275-8.

22. Wright TI, Baddour LM, Berbari EF, Roenigk RK, Phillips PK, Jacobs MA, et al. Antibiotic prophylaxis in dermatologic surgery: advisory statement 2008. J Am Acad Dermatol. 2008;59:464-73.

23. Dixon AJ, Dixon MP, Dixon JB, Del Mar CB. Prospective study of skin surgery in smokers vs. nonsmokers. Br J Dermatol. 2009;160:365-7.

24. Honaker JS, Bordeaux JS, Tuttle MS. Retrospective review of risk factors predictive of complications in patients after below the knee mohs micrographic surgery. Dermatol Surg. 2016;42:568-70.

25. Wahie S, Lawrence CM. Wound complications following diagnostic skin biopsies in dermatology inpatients. Arch Dermatol. 2007;143:1267-71.

26. Levender MM, Davis SA, Kwatra SG, Williford PM, Feldman SR. Use of topical antibiotics as prophylaxis in clean dermatologic procedures. J Am Acad Dermatol. 2012;66:445-51.

27. Khadim MF, Bell PR, Rashid A, Lewis HG. A postal survey of UK practice on discontinuation of anticoagulant/antithrombotics therapy before minor cutaneous surgery of the head and neck. J Plast Reconstr Aesthet Surg. 2011;64:e213-5.

28. Shimizu I, Jellinek NJ, Dufresne RG, Li T, Devarajan K, Perlis C. Multiple antithrombotic agents increase the risk of postoperative hemorrhage in dermatologic surgery. J Am Acad Dermatol. 2008;58:810-6.

29. Bordeaux JS, Martires KJ, Goldberg D, Pattee SF, Fu P, Maloney ME. Prospective evaluation of dermatologic surgery complications including patients on multiple antiplatelet and anticoagulant medications. J Am Acad Dermatol. 2011;65:576-83.

30. Bartlett GR. Does aspirin affect the outcome of minor cutaneous surgery? Br J Plast Surg. 1999:52:214-6.

31. Kargi E, Babuccu O, Hosnuter M, Babuccu B, Altinyazar C. Complications of minor cutaneous surgery in patients under anticoagulant treatment. Aesthetic Plast Surg. 2002;26:483-5.

32. Syed S, Adams BB, Liao W, Pipitone M, Gloster H. A prospective assessment of bleeding and international normalized ratio in warfarin-anticoagulated patients having cutaneous surgery. J Am Acad Dermatol. 2004:51:955-7.

33. Shalom A, Wong L. Outcome of aspirin use during excision of cutaneous lesions. Ann Plast Surg. 2003;50:296-8.

34. Billingsley EM, Maloney ME. Intraoperative and postoperative bleeding problems in patients taking warfarin, aspirin, and nonsteroidal antiinflammatory agents. A prospective study. Dermatol Surg. 1997;23:381-3; discussion 384-5.

35. Schanbacher CF, Bennett RG. Postoperative stroke after stopping warfarin for cutaneous surgery. Dermatol Surg. 2000;26:785-9.

36. Kovich O, Otley CC. Thrombotic complications related to discontinuation of warfarin and aspirin therapy perioperatively for cutaneous operation. J Am Acad Dermatol. 2003;48:233-7.

37. Alcalay J. Cutaneous surgery in patients receiving warfarin therapy. Dermatol Surg. 2001;27:756-8.

38. Goldminz D, Bennett RG. Cigarette smoking and flap and full-thickness graft necrosis. Arch Dermatol. 1991;127:1012-5.

39. Gill JF, Yu SS, Neuhaus IM. Tobacco smoking and dermatologic surgery. J Am Acad Dermatol. 2013;68:167-72.

40. Chan LK, Withey S, Butler PE. Smoking and wound healing problems in reduction mammaplasty: is the introduction of urine nicotine testing justified? Ann Plast Surg. 2006;56:111-5.

41. Kimyai-Asadi A, Goldberg LH, Peterson SR, Silapint S, Jih MH. The incidence of major complications from Mohs micrographic surgery performed in office-based and hospitalbased settings. J Am Acad Dermatol. 2005;53:628-34.

42. Hansen TJ, Lolis M, Goldberg DJ, MacFarlane DF. Patient safety in dermatologic surgery: part I. Safety related to surgical procedures. J Am Acad Dermatol. 2015;73:1-12; quiz 13-4.

43. Otley CC. Perioperative evaluation and management in dermatologic surgery. J Am Acad Dermatol. 2006;54:119-27. 